Article

\title{
Evaluating the death and recovery of lateral line hair cells following repeated neomycin treatments.
}

\author{
Alexandra Venuto ${ }^{1}$ and Timothy Erickson 1,2,* \\ 1 Department of Biology, East Carolina University, Greenville, NC, United States; venutoa18@stu- \\ dents.ecu.edu (A.V.) \\ 2 Department of Biology, University of New Brunswick, Fredericton, NB, Canada; timothy.erickson@unb.ca \\ (Current address, T.E.) \\ * Correspondence: timothy.erickson@unb.ca
}

\begin{abstract}
Acute chemical ablation of lateral line hair cells is an important tool to understand lateral line-mediated behaviors in free-swimming fish larvae and adults. However, lateral line-mediated behaviors have not been described in fish larvae prior to swim bladder inflation, possibly because single doses of ototoxin do not effectively silence lateral line function at early developmental stages. To determine if ototoxins can disrupt lateral line hair cells during early development, we repeatedly expose zebrafish larvae to the ototoxin neomycin during a 36-hour period from 3-4 days post-fertilization (dpf). We use simultaneous transgenic and vital dye labeling of hair cells to compare 6- hour and 12-hour repeated treatment timelines and neomycin concentrations between $0-400 \mu \mathrm{M}$ in terms of larval survival, hair cell death, regeneration, and functional recovery. Following exposure to neomycin, we find that the emergence of newly functional hair cells outpaces cellular regeneration, likely due to the maturation of ototoxin-resistant hair cells that survive treatment. Furthermore, hair cells of $4 \mathrm{dpf}$ larvae exhibit faster recovery compared to $3 \mathrm{dpf}$ larvae. Our data suggest that the rapid functional maturation of ototoxin-resistant hair cells limits the effectiveness of chemical-based methods to disrupt lateral line function. Furthermore, we show that repeated neomycin treatments can continually ablate functional lateral line hair cells between 3-4 dpf in larval zebrafish.
\end{abstract}

Keywords: zebrafish; lateral line; neuromast; hair cell; ototoxicity; toxicity; regeneration; cell death; neomycin; aminoglycosides

\section{Introduction}

Fish and some amphibians possess a unique sensory system called the mechanosensory lateral line, which is comprised of clusters of mechanically-sensitive hair cells distributed all over the body in organs called neuromasts. The lateral line detects hydrodynamic information and contributes to several behaviors such as foraging, predator avoidance, and swimming coordination [1,2]. For example, the lateral line is a critical sensory component for larval and adult fish to orient to water flow (rheotaxis) [3-5]. Additionally, aquatic organisms can identify locations of predators and prey by combining the direction and force of the water interacting with the neuromast pattern along the body $[6,7]$.

To investigate these lateral line-mediated behaviors, researchers use a variety of physical and chemical strategies to functionally disrupt the lateral line [8,9]. The use of ototoxins to chemically ablate hair cells is the most popular technique due to their specificity, convenience, and applicability to a wide range of aquatic vertebrates. Common ototoxins include aminoglycoside antibiotics (e.g. neomycin), certain heavy metals (e.g. copper), and various platinum derivatives (e.g. cisplatin) [10-13]. These compounds disrupt the lateral line by entering hair cells through functional mechanotransduction channels, causing oxidative stress, and triggering apoptotic cell death [14-18]. While useful for lateral line research, aminoglycosides are also major causes of irreversible hearing loss in 
humans [19]. Understanding how zebrafish hair cells respond to ototoxic assaults may lead to preventative or therapeutic strategies to combat human hearing loss [20].

The acute chemical ablation of hair cells is a valuable tool for studies on adult fish and free-swimming larvae. However, there is little information about how the lateral line influences behavior in early-stage larvae, likely because ototoxins are less effective at early developmental stages [21,22]. Previous research suggests two reasons why a single dose of ototoxin is less effective at younger stages compared to older larvae and adults: (i) the rapid proliferation of hair cells at early developmental stages, and (ii) the presence of immature hair cells that are resistant to chemical ablation. Regarding proliferation, initial neuromast deposition in developing zebrafish begins as early as 20 hours post-fertilization (hpf), and hair cells start to gain function by $34 \mathrm{hpf}$ [23-26]. The number of hair cells per neuromast continuously grows during 3-5 days post-fertilization (dpf) and results in the generation of over 300 functional hair cells [13,23,26,27]. Therefore, a single ototoxic treatment during this timeframe is unlikely to fully disable the lateral line, especially considering that approximately half of all hair cells are regenerated within 24 hours posttreatment [13]. It has been noted that regeneration at this stage of development is difficult to track due to the rapid growth of the lateral line [27]. Regarding ototoxin-resistance, susceptibility to chemical ablation requires that hair cells possess functional mechanotransduction channels [28]. Immature hair cells that have not yet developed mechanosensitivity are resistant to the effects of ototoxins [21,22], similar to hair cells where mechanotransduction is disabled through chemical or genetic means [29,30]. Developing neuromasts may contain a relatively high proportion of immature hair cells $[21,22,31]$ that can potentially gain mechanosensitivity after ototoxin treatment, allowing neuromasts to partially regain function without regenerating new hair cells. Indeed, following exposure to ototoxin, lateral line-mediated behaviors of zebrafish larvae recover before hair cell regeneration is complete [5,32]. However, whether functional maturation of immature hair cells precedes cellular regeneration has not been tested explicitly.

Recently, we reported the first genetic model for the specific loss of lateral line function in zebrafish [33]. To complement our studies with this mutant, here we aim to identify a timeline of repeated neomycin treatments in larval zebrafish aged 3-4 dpf that will mimic the congenital loss of lateral line function while minimizing off-target toxicity. We use two treatment timelines (every 6 or 12 hours) with neomycin concentrations from 50$400 \mu \mathrm{M}$. We compare these treatment regimens in terms of larval survival, efficacy of hair cell ablation, functional maturation of hair cells, and cellular regeneration of hair cells following repeated neomycin treatments. Our data supports the hypothesis that rapid functional maturation of ototoxin-resistant hair cells diminishes the effectiveness of ototoxins as a tool to study the lateral line. We also identify a set of treatment conditions that can be used in future studies along with the lateral line mutant to investigate how the lateral line contributes to the behaviors of larval zebrafish prior to $5 \mathrm{dpf}$.

\section{Results}

2.1. Toxicity of repeated neomycin treatments in 3-4 dpf zebrafish.

In the following experiments, we use repeated neomycin treatments delivered at either 6-hour or 12-hour intervals over the course of 36 hours in zebrafish at 3-4 dpf and at neomycin concentrations of $0,50,100,200,300$, and $400 \mu \mathrm{m}$ (Figure 1A). 


\section{A Timeline One: 6-hour treatment intervals}

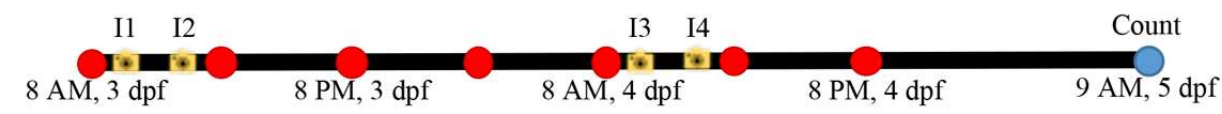

Timeline Two: 12-hour treatment intervals

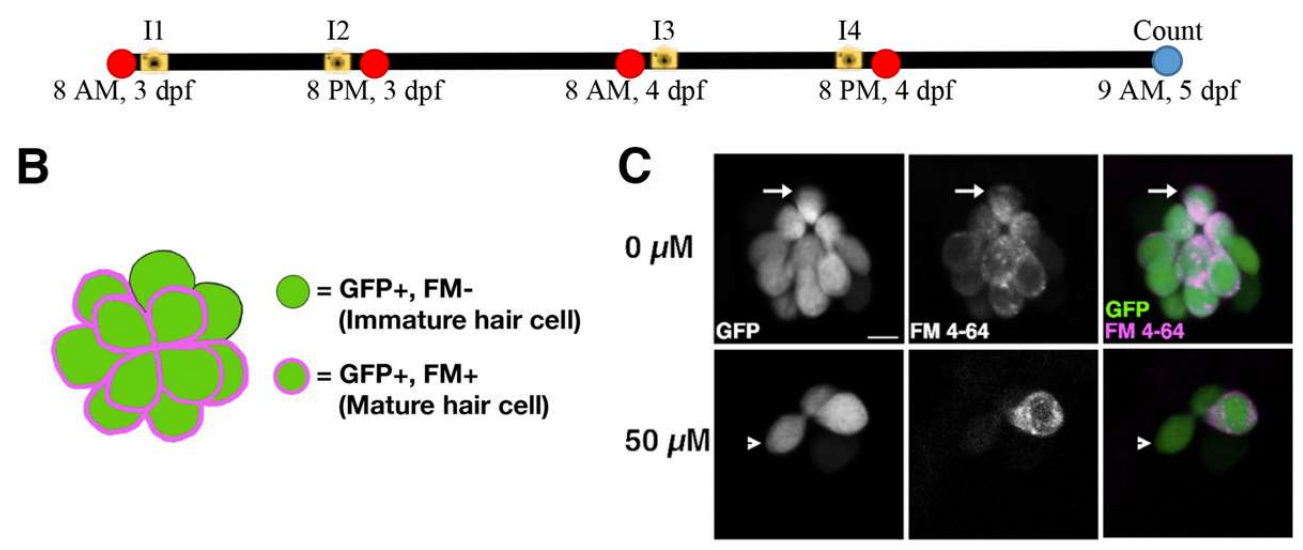

Figure 1. Experimental set-up. (A) The 6-hour and 12-hour treatment timelines with neomycin concentrations of 0, 50, 200, 300, and $400 \mu \mathrm{M}$ beginning $8 \mathrm{AM}$ at 3 days post-fertilization (dpf). Red dots indicate treatment timepoints, yellow cameras indicate imaging timepoints for hair cell counts (I1-I4), and blue dots indicate final count of surviving larvae. (B) Representation of a neuromast showing the strategy used to differentiate between mature and immature hair cells. Mature cells are labelled with both GFP and FM 4-64 while immature cells are labelled with GFP, but not FM 4-64. (C) Representative confocal images of O2 neuromasts at imaging timepoint I1 in the 0 and $50 \mu \mathrm{M}$ neomycin treatment groups. The Tg(myo6b:eGFP-pA)vo68Tg line is green and FM 4-64 is magenta in the merge of the two channels in the third column. The full arrow indicates an example of a functional hair cell that is positive for both GFP and FM 4-64. The arrowhead indicates an example of an ototoxin-resistant, immature hair cell that is positive for GFP only. Scale bar $=5 \mu \mathrm{m}$.

To assess general toxicity that may be associated with repeated neomycin treatments, we quantified survivorship across treatment schedules and concentrations. Neomycin concentrations of $100 \mu \mathrm{M}$ or higher result in greater than $50 \%$ larval mortality when administered every 6 hours (Figure 2A and Table S1). The two highest concentrations, 300 and $400 \mu \mathrm{M}$, result in $100 \%$ lethality and only the $50 \mu \mathrm{M}$ concentration allows for greater than $50 \%$ survival using the 6-hour treatment timeline. 12-hour treatment intervals result in improved survival relative to the 6-hour intervals. While only $18 \%$ of larvae survive when exposed to $400 \mu \mathrm{M}$ neomycin every 12 hours $(\mathrm{n}=64)$, survival was almost $50 \%$ at 200 and $300 \mu \mathrm{M}, 67 \%$ at the $100 \mu \mathrm{M}$, and $86 \%$ at $50 \mu \mathrm{M}$ (Figure 2A and Table S1). Despite increased survival using the 12-hour interval, many larvae from the $300 \mu \mathrm{M}$ and $400 \mu \mathrm{M}$ neomycin treatment groups exhibited pericardial edema and were smaller in size compared to untreated larvae at $5 \mathrm{dpf}(\mathrm{n}=6 / 30$ and $5 / 16$ in the 300 and $400 \mu \mathrm{m}$ groups, respectively) (Figure 2B-D). To determine which factors were culpable for the mortality rates, we ran a generalized linear model regressing percent survival after 24 hours against concentration of neomycin and the experimental timeline used (Table 1). Treatment concentrations $100 \mu \mathrm{M}$ and greater are significantly associated with increased mortality $(\mathrm{p}<0.0001)$, while less frequent treatments favors survival $(\mathrm{p}<0.0001)$. Overall, we find that repeated neomycin treatments at 3-4 dpf using concentrations over $50 \mu \mathrm{M}$ result in high levels of larval mortality. 


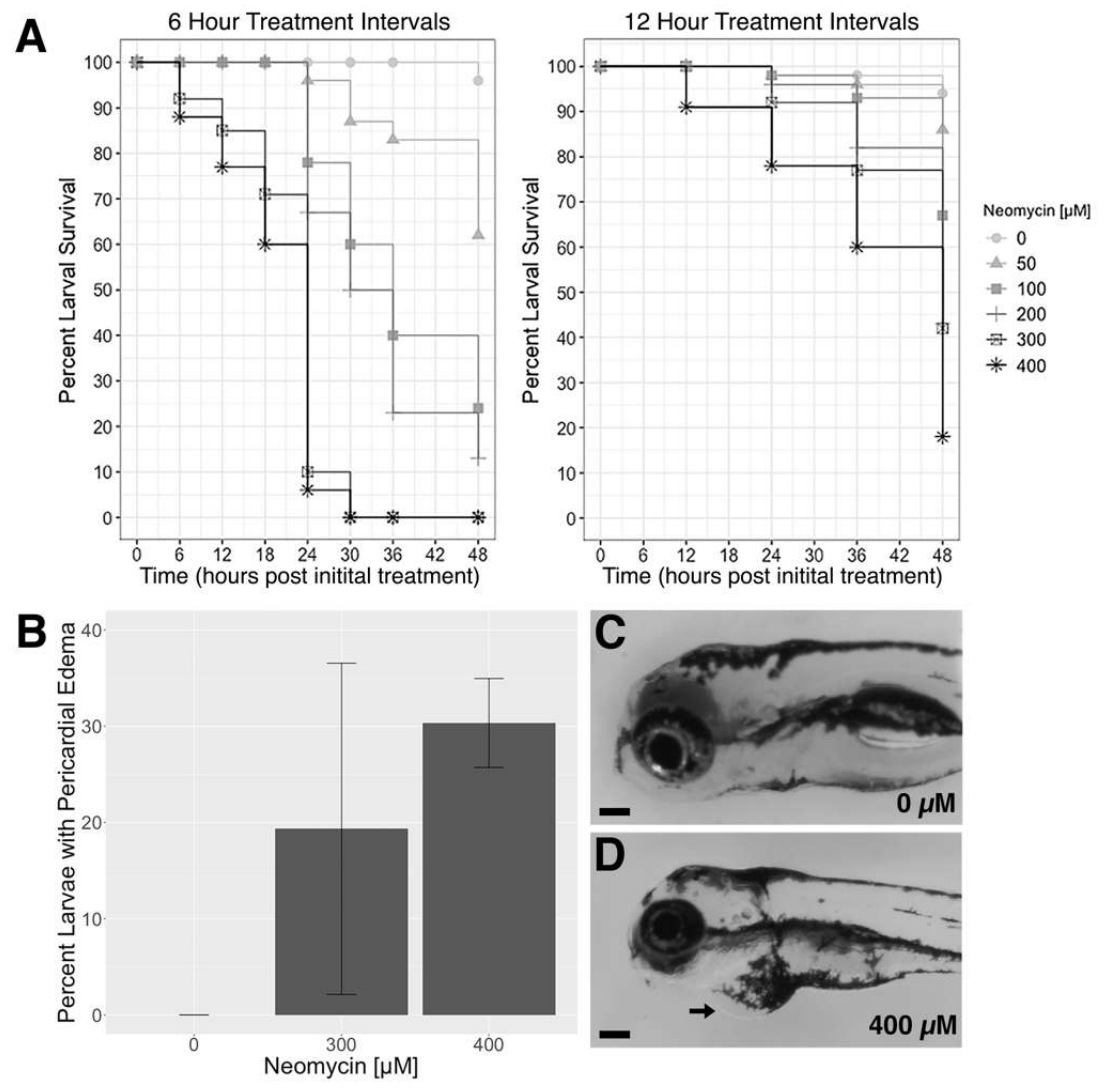

Figure 2. Toxicity of repeated neomycin treatments in zebrafish larvae. (A) Percent survival for Timeline One (6-hour treatment intervals) and Timeline Two (12-hour intervals). Final counts were done at $5 \mathrm{dpf}, 48$ hours following the first treatment. (B) Average percent of larvae with pericardial edema at 5 days post-fertilization (dpf) after four neomycin treatments given at 12-hour intervals. Error bars represent standard deviation. (C) Image of an untreated ( $0 \mu \mathrm{M}$ neomycin) larva at $5 \mathrm{dpf}$. (D) Image of a $5 \mathrm{dpf}$ larva exhibiting pericardial edema (arrow) after 4 treatments with $400 \mu \mathrm{M}$ neomycin delivered every 12 hours. Scale bar $=0.1 \mathrm{~mm}$.

Table 1. Results of a beta regression model regressing percent survival at 24 hours against neomycin concentration and treatment timeline to show which variables are significantly contributing to larval survival or mortality $(* * * * p<0.0001$, ns $=$ not significant).

\begin{tabular}{lcccc}
\hline \multicolumn{1}{c}{ Beta } & Estimate & $\begin{array}{c}\text { Standard } \\
\text { Error }\end{array}$ & P value & $\begin{array}{c}\text { Significance } \\
\text { Level }\end{array}$ \\
\hline Intercept $(0 \mu \mathrm{M}, 6$ Hours $)$ & 4.1322996 & 0.4913248 & $4.08 \mathrm{E}-17$ & $* * * *$ \\
$50 \mu \mathrm{M}$ Neomycin & -0.9457874 & 0.5789734 & 0.102 & $\mathrm{~ns}$ \\
$100 \mu \mathrm{M}$ Neomycin & -2.6188895 & 0.5660887 & $3.72 \mathrm{E}-06$ & $* * * *$ \\
$200 \mu \mathrm{M}$ Neomycin & -3.2026104 & 0.5625068 & $1.24 \mathrm{E}-08$ & $* * * *$ \\
$300 \mu \mathrm{M}$ Neomycin & -5.2428102 & 0.6140916 & $1.37 \mathrm{E}-17$ & $* * * *$ \\
$400 \mu \mathrm{M}$ Neomycin & -5.8515287 & 0.6317583 & $2.00 \mathrm{E}-20$ & $* * * *$ \\
12 Hour Treatment Intervals & 2.5645373 & 0.3409579 & $5.41 \mathrm{E}-14$ & $* * * *$ \\
\hline
\end{tabular}




\subsection{Strategy to differentiate between mature and immature hair cells.}

A single neomycin treatment can acutely disable the lateral line in zebrafish $5 \mathrm{dpf}$ and older [13,32], but is less effective on larvae younger than $5 \mathrm{dpf}$, possibly due to the presence of immature hair cells that are resistant to ototoxins [21,22]. To test this hypothesis, we utilize simultaneous transgenic and vital dye labeling of lateral line hair cells to distinguish between mature, mechanically-sensitive hair cells and immature hair cells that have yet to develop a functional transduction apparatus (Figure 1B, C). We mark both immature and mature hair cells using the Tg(myo6b:eGFP-pA)vo68Tg line [33], which uses the myo6b promoter to drive GFP in all hair cells beginning at an early stage of their development [34]. Simultaneously, we mark functional hair cells with FM 4-64, a far-red fluorescent dye that rapidly enters hair cells through functional mechanotransduction channels $[30,35]$. In this study, we define mature hair cells as being positive for both GFP and FM 4-64 and define immature hair cells as being GFP-positive but FM 4-64 negative. In this way, we can compare the proportion of functional cells to total hair cells, evaluate the efficacy of neomycin treatment, and quantify the functional and cellular regeneration of the lateral line following repeated neomycin treatments.

\subsection{Hair cell proliferation and maturation in untreated larvae.}

Chemical ablation of lateral line function may be less effective in larvae younger than $5 \mathrm{dpf}$ due to the natural proliferation of hair cells at this developmental stage. To determine if neuromasts are still adding new hair cells between 3-4 dpf, we quantified the total number of hair cells (GFP+) and the number of functional hair cells (FM+) per neuromast in untreated larvae and expressed these numbers as a percent of the total number of GFP+ hair cells from the beginning of the experiment. There is continuous cell proliferation and functional maturation of lateral line hair cells during the 36-hour period between imaging timepoints I1 and I4 in untreated larvae (Figure 3 and $0 \mu \mathrm{m}$ neomycin rows in Table S2). On average, neuromasts add 1.6 GFP-positive hair cells between imaging timepoints 1 and 4, representing a 12.0\% increase during these 36 hours of development. Over the same period, neuromasts add an average of two FM-positive cells, a 18.9\% increase (Figure 3 and Table S2). The number of functional, FM-positive hair cells is consistently less than the GFP-positive hair cells at all imaging timepoints, yet there is an overall increase in the the proportion of FM-positive cells relative to the total number of hair cells per neuromast between imaging timepoints I1 and I4 (FM / GFP $=0.849$ for I1, 0.995 for I4; $\mathrm{z}$-score test for two proportions, $\mathrm{z}=-2.31, \mathrm{p}=0.021$ ) (Figure 3). This indicates that a greater proportion of hair cells in each neuromast are mechanically-sensitive as development procedes. Together, these data suggest that, between days 3-4 of zebrafish development, the number of hair cells per neuromast has not yet reached steady-state and both nascent and mature hair cells are still being added. 


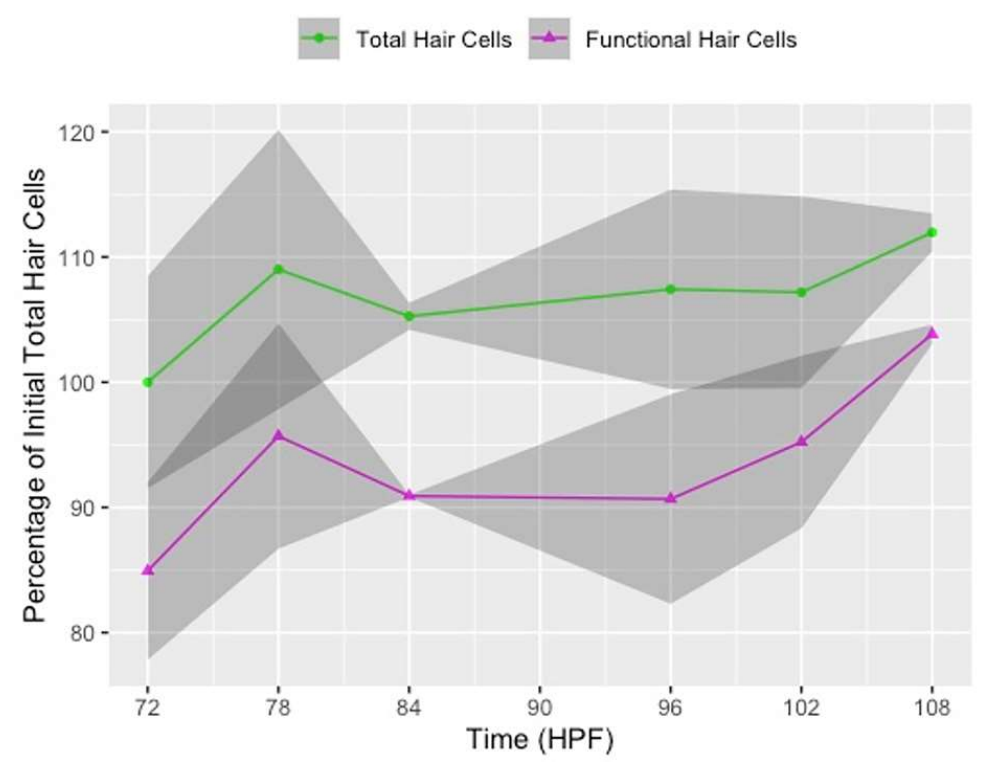

Figure 3. Hair cell proliferation and maturation in untreated $(0 \mu \mathrm{M}$ neomycin) larvae between 3-4 days post-fertilization (dpf). Line graph of the percentage of total (GFP+, green line) and functional (FM 4-64+, magenta line) hair cells in untreated larvae relative to the total number of GFP+ hair cells when larvae were $72 \mathrm{hpf}(3 \mathrm{dpf})$ at the beginning of the experiment. Data points are from each imaging timepoint from all control group replicates of the 6- and 12-hour timelines combined. The x-axis represents hours postfertilization (HPF). The shaded regions surrounding the line represent the $95 \%$ confidence intervals. Average counts are provided in the $0 \mu \mathrm{M}$ neomycin rows of Table S2 and counts from individual neuromasts in the 12-hour timeline are provided in Figure S1.

\subsection{Effect of neomycin concentration and treatment interval on hair cell death.}

To measure the effect of neomycin concentration and treatment interval on the efficacy of hair cell ablation, we quantified the number of GFP-positive and FM-positive hair cells that remain following neomycin exposure at imaging time points I1 and I3. We excluded the 300 and $400 \mu \mathrm{M}$ neomycin treatments from these analyses due to high mortality and abnormal morphology observed at those concentrations (Figure 2). Consistently, we find that GFP-positive, FM-negative hair cells remain after a 25-minute exposure to neomycin at concentrations ranging from $50-200 \mu \mathrm{M}$ (Figure $4 \mathrm{~A}$ ). At imaging timepoints 1 and 3, neomycin concentration did not significantly affect the number of remaining GFP-positive cells using either 6- or 12- hour treatment intervals (ANOVA with Dunn post-test, $\alpha=0.05$ ) (Figure $4 \mathrm{~A}$, Table S3). Next, looking only at mechanicallysensitive (FM-positive) hair cells, neomycin concentration did not have a significant effect on the number of FM-positive cells remaining after treatment at imaging timepoint 1 in either the 6- or 12- hour treatment intervals, with the exception of the comparison between the 50 and $200 \mu \mathrm{M}$ neomycin concentrations (ANOVA with Dunn post-test, $\mathrm{p}=0.042$ ) (Figure 4B, Table S3). At imaging timepoint 3 within both the 6- and 12-hour treatment experiments, neomycin concentration did not have a significant effect on the number of FM-positive cells remaining after treatment (ANOVA with Dunn post-test, $\alpha=0.05$ ) (Figure 4B). However, we do observe a trend towards decreasing numbers of total and functional hair cells as neomycin concentration increases (Figure 4). 

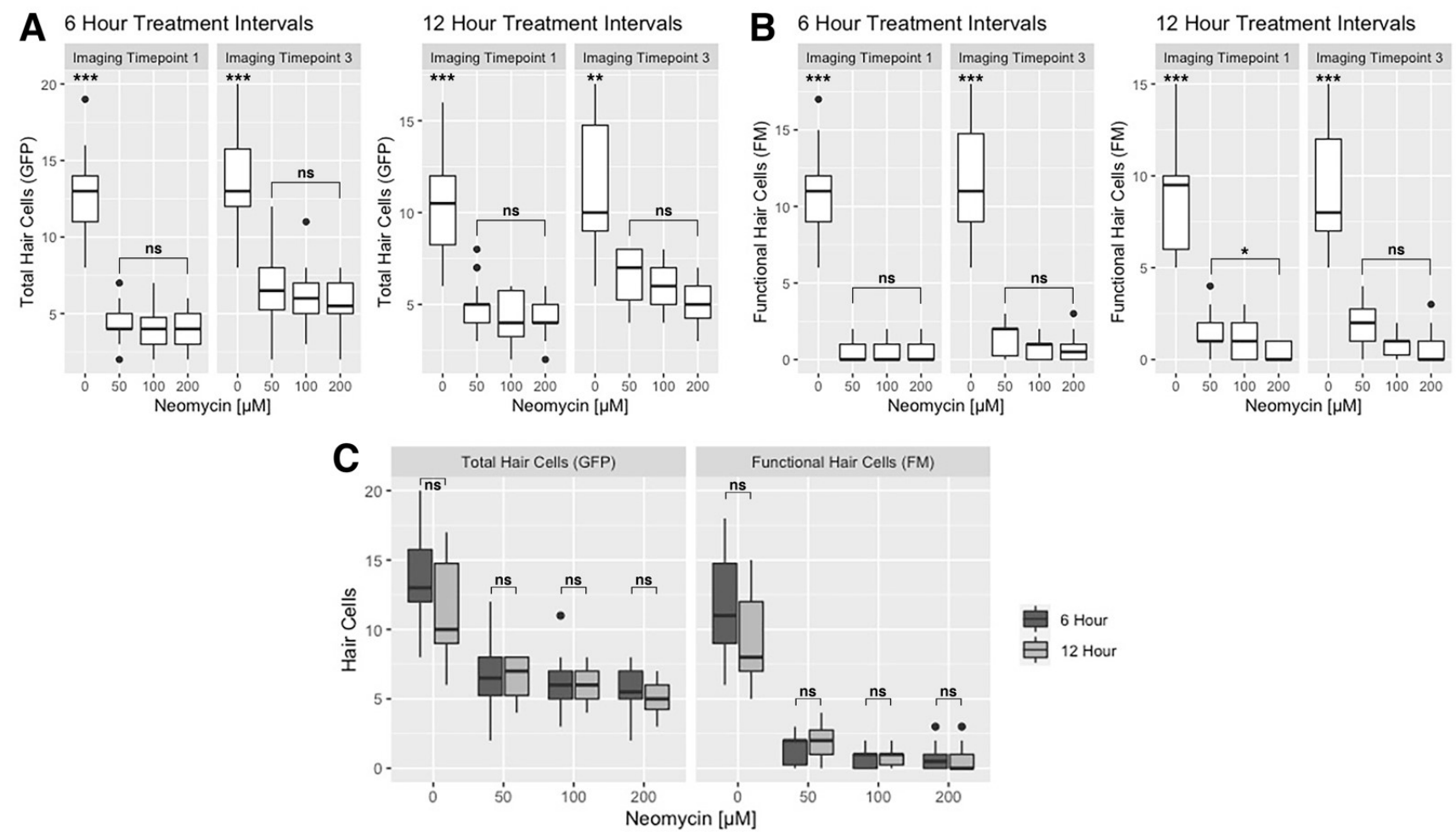

Figure 4. Effect of neomycin concentration and treatment intervals on hair cell death. Box plots represent the number of hair cells per neuromast for both the 6-hour and 12-hour treatment timelines. (A) Total (GFP+) and (B) functional (FM+) hair cells at imaging timepoints I1 and I3. The Kruskal-Wallis ANOVA with Dunn post-test results are provided in Table S3. (C) Comparison of the 6hour and 12-hour timelines in terms of the number of GFP+ and FM+ hair cells remaining after treatment at imaging timepoint I3 on $4 \mathrm{dpf}$. There is no significant difference in the hair cell counts between the two treatment timelines (Kruskal-Wallis ANOVA with Dunn post-test, $\alpha=0.05$; Table S4). Significance levels are as follows: ${ }^{* * *}=p<0.001,{ }^{* *}=p<0.01,{ }^{*}=p<0.05$, ns $=$ not significant.

Next, we analyzed whether the frequency of neomycin treatments had an effect on the level of hair cell ablation by comparing the 6- and 12-hour timelines in terms of the difference in the number of total and functional hair cells at imaging timepoint 3 for all neomycin concentrations. We find that more frequent treatments do not have a significant effect on cell death (ANOVA with Dunn post-test, $\alpha=0.05$ ) (Figure 4C, Table S4). Overall, we find that mechanically-sensitive hair cells in zebrafish at age 3-4 dpf are susceptible to neomycin exposure and can be continuously disrupted over 36 hours. Furthermore, neuromasts from 3-4 dpf larvae contain immature, neomycin-resistant hair cells that remain after treatment.

\subsection{Cellular and functional regeneration in 3 dpf larval zebrafish.}

We assessed cellular and functional regeneration following exposure to neomycin in 3 dpf larvae by analyzing the data from imaging timepoints I1 and I2. Given the high mortality rates associated with repeated neomycin treatments of $100-400 \mu \mathrm{M}$ (Figure 2), we focused our analysis on the $50 \mu \mathrm{M}$ condition. We observe a significant increase in the number of functional (FM-positive) hair cells between time points I1 and I2 for both the 6- and 12-hour treatment intervals (Welch's t-test, 6-hour: $\mathrm{M}=0.8, \mathrm{SD}=0.4, \mathrm{p}=0.028$; 12 hour: $\mathrm{M}=1.0, \mathrm{SD}=0.3, \mathrm{p}=0.002$ ) (Figure $5 \mathrm{~A}$ ). There was no significant change in the number of GFP-positive cells between time points I1 and I2 in either the 6- or 12-hours experiments (6-hour: $\mathrm{M}=0.39, \mathrm{SD}=0.71, \mathrm{p}=0.407$; 12-hour: $\mathrm{M}=0.56, \mathrm{SD}=0.16, \mathrm{p}=0.319$ ) (Figure 5A). We conclude that the surviving immature hair cells start to acquire mechanosensitivity within 6 to 12 hours after neomycin treatment and that functional maturation of hair cells outpaces the regeneration of new hair cells at $3 \mathrm{dpf}$. 
A 6 Hour Treatment Intervals

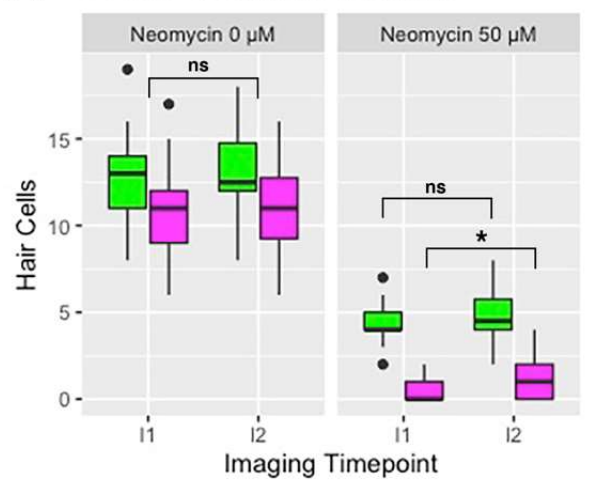

B 6 Hour Treatment Intervals

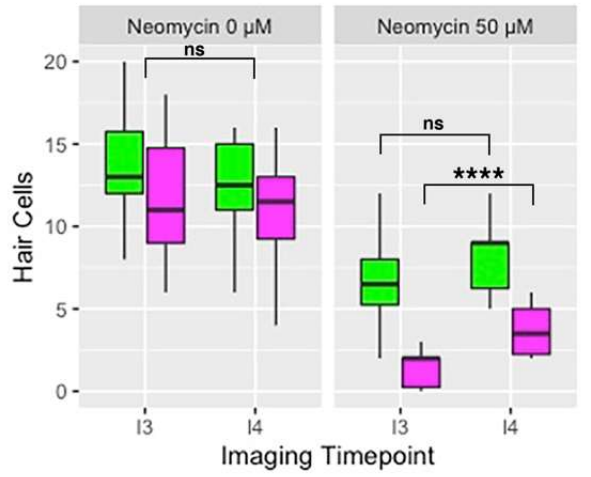

12 Hour Treatment Intervals

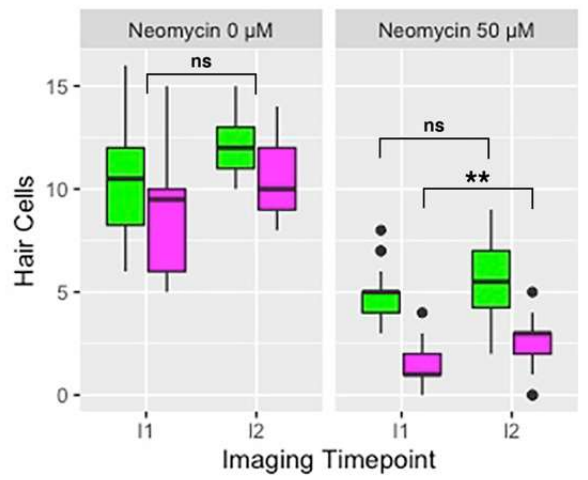

官 Total Hair Cells
宁 Functional Hair Cells

12 Hour Treatment Intervals

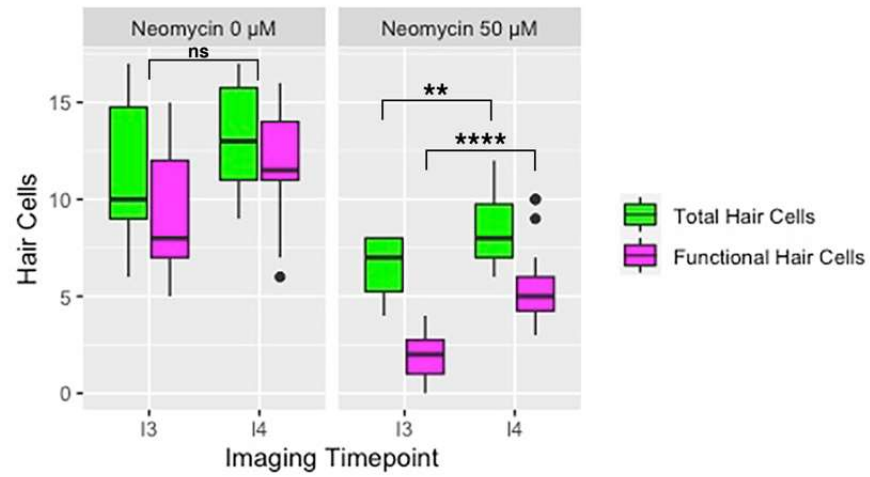

Figure 5. Comparison of hair cell counts in the $0 \mu \mathrm{M}$ and $50 \mu \mathrm{M}$ neomycin treatment groups at (A) $3 \mathrm{dpf}$ and (B) $4 \mathrm{dpf}$. Green box plots indicate cell counts using the GFP transgene to mark both transducing and non-transducing hair cells and the magenta box plots indicate hair cell counts using FM 4-64 to label mechanically-sensitive hair cells. See Figure 1A for timing of imaging timepoints I1-I4. Performed Kruskal-Wallis ANOVA with Dunn post-test to find significance levels, which are as follows: ${ }^{* * *}=\mathrm{p}<0.0001,{ }^{* *}=$ $\mathrm{p}<0.01,{ }^{*}=\mathrm{p}<0.05, \mathrm{~ns}=$ not significant.

\subsection{Cellular and functional regeneration in 4 dpf larval zebrafish.}

Like the analyses done at $3 \mathrm{dpf}$, we assessed cellular and functional recovery at $4 \mathrm{dpf}$ using $50 \mu \mathrm{M}$ neomycin given every 6 or 12 hours. Between imaging time points I3 and I4, we observe a significant addition of FM-positive hair cells between 6-hour treatments and 12-hour treatments at $4 \mathrm{dpf}$ (Welch's t-test, 6-hour: $\mathrm{M}=2.2, \mathrm{SD}=0.9, \mathrm{p}=0.000016$; 12-hour: $\mathrm{M}=3.9, \mathrm{SD}=0.2, \mathrm{p}<0.00001$ ) (Figure $5 \mathrm{~B}$ ). In the 6-hour treatment timeline, there is no significant increase in the number of GFP-positive hair cells between imaging timepoints at $4 \mathrm{dpf}(\mathrm{M}=1.3, \mathrm{SD}=1.1, \mathrm{p}=0.125)$. However, in the 12-hour treatment interval, there is a significant increase in the number of GFP-positive hair cells between I3 and I4 at $4 \mathrm{dpf}$ $(\mathrm{M}=1.6, \mathrm{SD}=0.5, \mathrm{p}=0.004)$ (Figure $5 \mathrm{~B})$. These data suggest that, after neomycin treatment at $4 \mathrm{dpf}$, the remaining immature hair cells start to gain functionality within 6 hours while the regeneration of new hair cells begins within 12 hours. 


\subsection{Comparison of hair cell death and recovery between 3 and 4 dpf zebrafish.}

Next, we compared the susceptibility of hair cells to $50 \mu \mathrm{M}$ neomycin at $3 \mathrm{dpf}$ and 4 dpf by quantifying the ratio of functional ( $\mathrm{FM}+$ ) to total (GFP+) hair cells from imaging timepoints I1 (3 dpf) and I3 (4 dpf) for both timelines. Comparing I1 and I3, the proportions of functional hair cells per neuromast that remain following treatment are not significantly different in the 6-hour or 12-hour treatment timelines (z-score test for two proportions, 6-hour: $\mathrm{z}=-1.01, \mathrm{p}=0.313,12$-hour: $\mathrm{z}=-0.38, \mathrm{p}=0.704$ ) (Figure 6). Even though it is not statistically significant, there is a consistent increase in the ratio of FM:GFP from I1 to I3 in both timelines. To determine which factors were responsible for these results, we ran a generalized linear model regressing hair cell proportions against the days post fertilization and the number of treatments (Table 2). According to this beta-regression model, transitioning from $3 \mathrm{dpf}$ to $4 \mathrm{dpf}$ is associated with an increase in the proportion of mechanically-sensitive $(\mathrm{FM}+)$ hair cells per neuromast $(\mathrm{p}<0.001)$. Conversely, the number of neomycin treatments are significantly associated with a decrease in the proportion of mechanically-sensitive cells $(\mathrm{p}<0.001)$. Overall, we find that the proportions of functional hair cells that remain after neomycin treatment are statistically similar between 3 and $4 \mathrm{dpf}$, possibly due to the opposing effects of developmental time and repeated exposures to neomycin.

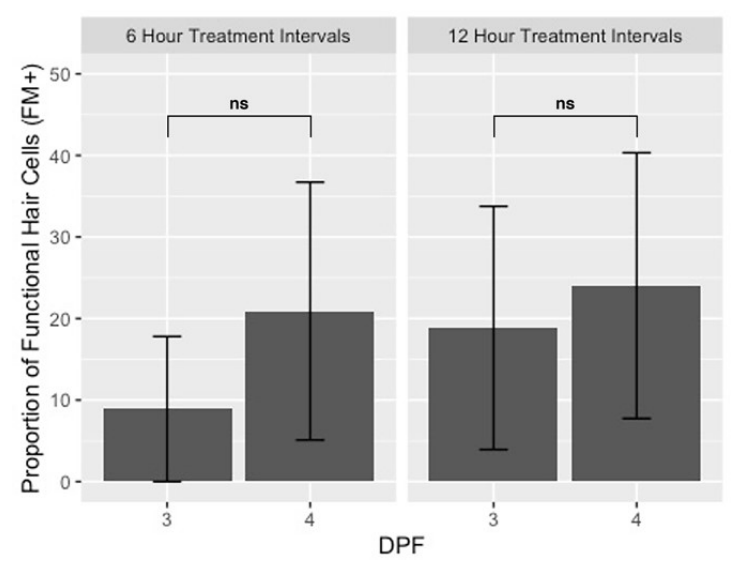

Figure 6. Bar graph showing the proportion of functional hair cells (FM+) relative to total hair cells (GFP+) one hour after $50 \mu \mathrm{M}$ neomycin treatments on 3 days post-fertilization (dpf) (I1) and $4 \mathrm{dpf}$ (I3) for both 6-hour and 12-hour timelines. Error bars represent standard deviation. Z-score test for proportions was used to evaluate the difference between the proportion of hair cells at 3 and 4 dpf. ns = not significant.

Table 2. Results of a beta regression model regressing FM:GFP hair cell proportions against the days post fertilization (dpf), treatment timeline, and number of treatments to show which factors were significant in contributing to the observed proportions of $\mathrm{FM}+\mathrm{GFP}+$ hair cells between 3 and $4 \mathrm{dpf}(* * *=\mathrm{p}<0.001$, ns $=$ not significant $)$.

\begin{tabular}{lcccc}
\hline \multicolumn{1}{c}{ Beta } & Estimate & $\begin{array}{c}\text { Standard } \\
\text { Error }\end{array}$ & P value & $\begin{array}{c}\text { Significance } \\
\text { Level }\end{array}$ \\
\hline Intercept $(0 \mu \mathrm{M}, \mathrm{I} 1)$ & 0.1024 & 0.1330 & 0.441 & $\mathrm{~ns}$ \\
$4 \mathrm{dpf}$ & 1.1435 & 0.2106 & $5.68 \mathrm{E}-8$ & $* * *$ \\
Number of Treatments & -0.6593 & 0.0655 & $<2 \mathrm{E}-16$ & $* * *$ \\
\hline
\end{tabular}

To assess differences in hair cell proliferation and recovery between 3 and $4 \mathrm{dpf}$, we compared the change in the number of hair cells between imaging timepoints I1 and I2 (3 $\mathrm{dpf})$ versus the change between I3 and I4 (4 dpf). There is a significant difference in the number of functional, FM+ hair cells added per neuromast after allowing for hair cell recovery between treatments at $4 \mathrm{dpf}$ compared to $3 \mathrm{dpf}$ in both the 6-hour and 12-hour 
treatment timelines (Welch's t-test, 6-hour: $\mathrm{p}=0.007$; 12-hour: $\mathrm{p}=0.00009$ ) (Figure 7A). There is no significant difference in the total number of GFP+ hair cells added per neuromast between treatments on $4 \mathrm{dpf}$ compared to $3 \mathrm{dpf}$ (6-hour: $\mathrm{p}=0.381$; 12-hour: $\mathrm{p}$ $=0.113$ ) (Figure 7B). Additionally, there is no significant difference in the recovery ratio of functional (FM+) to total (GFP+) hair cells between 3 and $4 \mathrm{dpf}$ in either treatment timeline (z-score test for two proportions, 6-hour: $\mathrm{z}=-0.93, \mathrm{p}=0.352,12$-hour: $\mathrm{z}=-1.96, \mathrm{p}=0.51$ ) (Figure 7C). Overall, we find that functional recovery of lateral line hair cells occurs more quickly at $4 \mathrm{dpf}$ compared to $3 \mathrm{dpf}$ in zebrafish larvae.
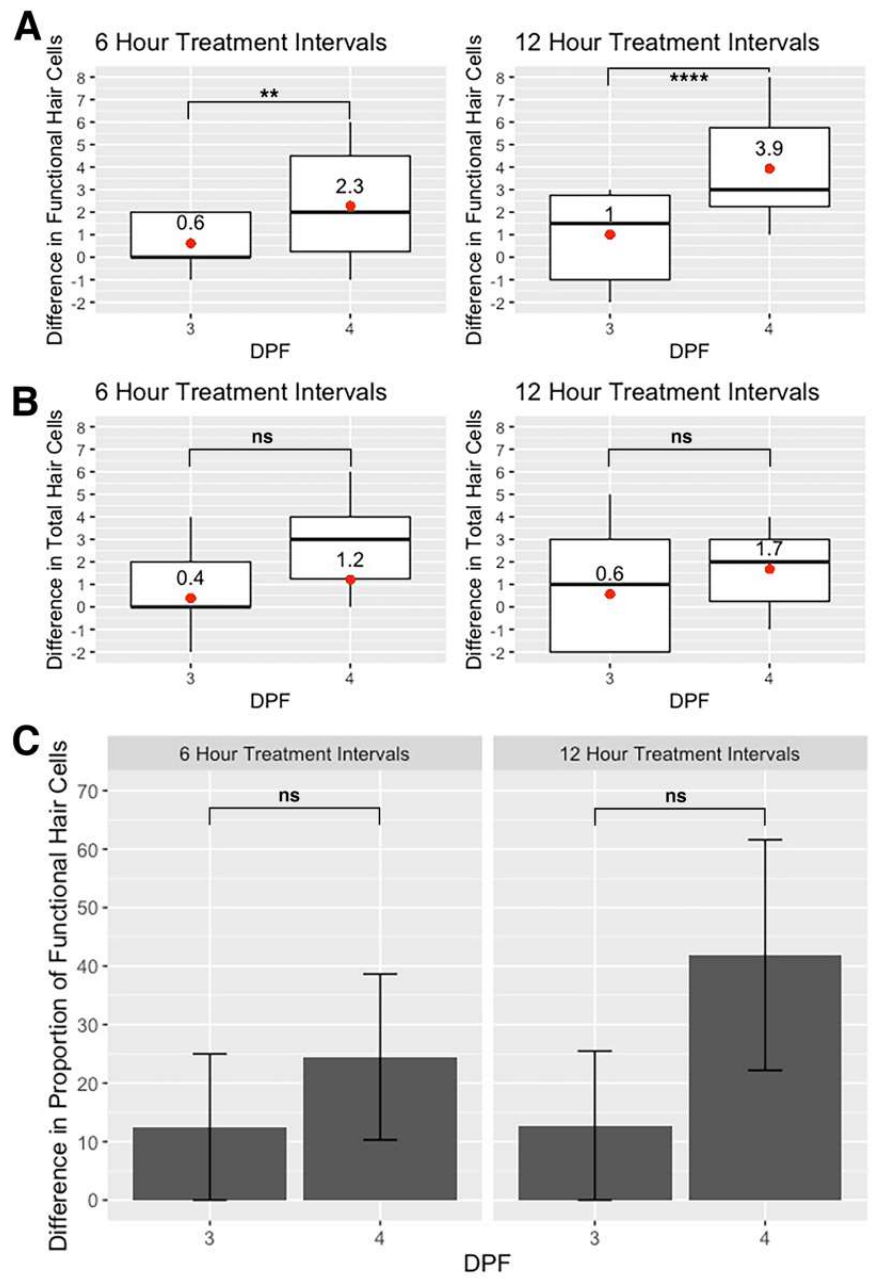

Figure 7. Hair cell recovery at 3 and 4 days post-fertilization (dpf). (A) Box plots showing the difference in functional (FM+) and (B) total (GFP+) hair cells between the first and second $50 \mu \mathrm{M}$ neomycin treatments on $3 \mathrm{dpf}$ (I2 minus I1) and $4 \mathrm{dpf}$ (I4 minus I3) in 6and 12-hour treatment timelines. Red dots and text indicate mean values. Statistal analysis was performed using Kruskal-Wallis ANOVA with Dunn post-test. (C) Bar graph showing the difference in the ratio of functional hair cells (FM+) to total hair cells (GFP+) between the first and second treatments on $3 \mathrm{dpf}$ (I2 minus I1) and $4 \mathrm{dpf}$ (I4 minus I3) for $50 \mu \mathrm{M}$ treatments in 6-hour and 12-hour treatment timelines. Z-score test for proportions was used to evaluate significance. Error bars represent standard deviation. Significance levels are as follows: ${ }^{* * *}=p<0.0001,{ }^{* *}=p<0.01$, ns $=$ not significant.

\section{Discussion}

In this study, we use repeated neomycin treatments to continuously ablate sensory hair cells of the lateral line in 3-4 dpf zebrafish larvae. We find that immature, ototoxinresistant hair cells remain after neomycin treatment, even after multiple exposures delivered every 6- or 12-hours. Our evidence suggests that these immature hair cells gain mechanosensitivity during the inter-treatment intervals, and that functional maturation of 
nascent hair cells occurs more rapidly at $4 \mathrm{dpf}$ compared to $3 \mathrm{dpf}$. To our knowledge, this is the first study to analyze hair cell death and recovery using repeated ototoxin treatments on 3-4 dpf zebrafish larvae. Overall, repeated neomycin treatments can be used to continuously disrupt mechanically-sensitive hair cells of the lateral line at this early stage of zebrafish development.

\subsection{Toxicity associated with repeated neomycin treatments.}

Single exposures to ototoxins are accepted to be well tolerated by larval and adult fish, thereby allowing their use in studies of lateral line-mediated behaviors [8]. However, our data clearly demonstrates that repeated neomycin treatments are toxic to larval zebrafish. Survival was less than $50 \%$ in the $100-400 \mu \mathrm{M}$ neomycin groups treated every 6 hours and the 200-400 $\mu \mathrm{M}$ neomycin groups treated every 12 hours. Furthermore, larval death occurred more rapidly with increased number and frequency of treatments (Figure 2). We consistently observe a major decline in larval survival between treatments four and five. This trend was apparent in both experimental timelines, despite differing treatment intervals. In the $300 \mu \mathrm{M}$ and $400 \mu \mathrm{M}$ neomycin treatment groups, surviving larvae often exhibit pericardial edema and developmental delays. Our findings are consistent with other studies that have demonstrated off-target toxicity of compounds commonly used in lateral line research [36-40]. Therefore, we conclude there is a toxicity threshold for larval zebrafish at 3-4 dpf that is surpassed after four treatments when using neomycin concentrations $100 \mu \mathrm{M}$ and above.

\subsection{Susceptibility of lateral line hair cells to repeated neomycin treatments at 3-4 dpf.}

Hair cells of the lateral line are rapidly proliferating and maturing at 3-4 dpf $[13,26,27]$. Mackenzie and Raible (2012) reported an increase in the number of functional hair cells per neuromast between 3-4 dpf and that the number of functional hair cells is significantly less than the total number of hair cells per neuromast at $5 \mathrm{dpf}$. At 3-4 dpf, we similarly find that the total complement of hair cells within a neuromast is always greater than the number of functional hair cells. Between 3-4 dpf, neuromasts in untreated larvae exhibit a $12 \%$ gain in total cells and $19 \%$ increase in functional cells over the same period. These results indicate that, as development proceeds, an increased proportion of hair cells per neuromast are mechanically-sensitive and therefore potentially susceptible to ototoxins. Furthermore, we find that these mechanically-sensitive hair cells are susceptible to neomycin in 3-4 dpf zebrafish and, in agreement with previous studies, that hair cell loss is incomplete due to functionally immature hair cells being resistant to the ototoxic effects of neomycin (Figures 4 and 5) [21,22,27,31].

\subsection{Hair cell maturation and regeneration after repeated neomycin treatments at 3-4 dpf.}

Previous studies have provided support for hair cell regeneration occurring more quickly in young larvae than in older larvae or adult fish $[27,41,42]$. In our study, we observe that there is a significant addition of functional hair cells within 6 hours post-treatment at both 3 and $4 \mathrm{dpf}$. The increase in functional hair cells outpaces the addition of new GFP+ hair cells at both 3 and $4 \mathrm{dpf}$. This is likely due to the functional maturation of immature GFP+ hair cells remaining after treatment. However, we found that the addition of both functional and total hair cells occurred more quickly at $4 \mathrm{dpf}$ than $3 \mathrm{dpf}$. Using the $50 \mu \mathrm{M}$ neomycin treatments, the ratio of functional to total (FM+/GFP+) hair cells increases by approximately $12 \%$ in 6 hours and 13\% in 12 hours on $3 \mathrm{dpf}$. On $4 \mathrm{dpf}$, the proportion of functional to total (FM+/GFP+) hair cells increases by approximately $25 \%$ in 6 hours and $42 \%$ in 12 hours. We saw that in untreated larvae, the number of hair cells per neuromast increases from $3 \mathrm{dpf}$ to $4 \mathrm{dpf}$. Therefore, the rate of functional and total hair cell addition following neomycin treatment at $4 \mathrm{dpf}$ may be quicker than at $3 \mathrm{dpf}$ due to a natural increased pace of hair cell proliferation. To confirm this, further studies investigating hair cell proliferation at 3 and $4 \mathrm{dpf}$ are necessary. 


\subsection{Mimicking the chronic loss of lateral line function in larval zebrafish at 3-4 dpf.}

To our knowledge, only one study has used repeated ototoxic treatments to study the long-term loss of lateral line-mediated behaviors [43]. However, this study was conducted on fish older than $5 \mathrm{dpf}$, and there may be lateral line-mediated behaviors in younger larvae (prior to swim bladder inflation) that have not been investigated. Given that even partial regeneration can restore lateral line function and behaviors [5,32,42], a single exposure to ototoxin is not an effective way to assess such behaviors in early-stage larvae when hair cells are actively proliferating and maturing. Therefore, repeated treatments appear necessary, but the feasibility of multiple treatments during lateral line formation has not been previously tested.

Our results support using $50 \mu \mathrm{M}$ neomycin every 12 hours to continuously disrupt hair cells of the lateral line in 3-4 dpf zebrafish larvae. Poor survivorship precludes the use of repeated neomycin treatments at concentrations of $100 \mu \mathrm{M}$ and greater, particularly when delivered every 6 hours (Figure 2). Furthermore, neomycin concentrations between $50-400 \mu \mathrm{M}$ are similar in their ability to kill mechanically-sensitive hair cells (Figure 4), suggesting there is little benefit to using higher concentrations. Although there is a statistically significant addition of functional hair cells between treatments for both the 6and 12-hour intervals, we observe little to no cellular regeneration (additional GFP+ hair cells) during the same intervals. Considering these results, and because rheotaxic behaviors begin to recover approximately 12 hours after exposure to neomycin at $5 \mathrm{dpf}$ [32], we advise treating 3-4 dpf larvae with $50 \mu \mathrm{M}$ neomycin every 12 hours to continuously disrupt lateral line hair cells while minimizing toxic side effects. This treatment regimen may complement the $l h f p l 5 b$ zebrafish mutant in analysis of lateral linemediated behaviors in early-stage larvae [33].

\section{Materials and Methods}

\subsection{Ethics statement and animal husbandry}

Animal research complied with guidelines stipulated by the Institutional Animal Care and Use Committee at East Carolina University (Greenville, NC, United States). Zebrafish (Danio rerio) were maintained and bred using standard procedures [44].

\subsection{Neomycin Sulfate Treatments}

A $1 \mathrm{mM}$ Neomycin sulfate (EMD Millipore Corp., USA) stock solution was made in $25 \mathrm{~mL}$ of E3 (embryo medium) and diluted in $35 \times 10 \mathrm{~mm}$ Petri dishes containing $30 \mathrm{~mL}$ E3 to make 50, 100, 200, 300 and $400 \mu \mathrm{M}$ concentrations for each treatment round. Timeline One involved neomycin treatments every six hours for a total of seven treatments while Timeline Two entailed treatments every twelve hours for a total of four treatments (Figure 1A). Both timelines were repeated three times at all neomycin concentrations. All treatments were done in Petri dishes containing 18-25 larvae and incubated for 25 minutes at $28.5^{\circ} \mathrm{C}$ before being returned to Petri dishes with fresh E3.

\subsection{Survivorship counts}

Starting with the first treatment at $8 \mathrm{AM}$ on $3 \mathrm{dpf}$, the number of surviving larvae in each treatment condition was recorded every 6 hours until the final treatment at $8 \mathrm{PM}$ on $4 \mathrm{dpf}$. The final survivorship count was made the following morning at $9 \mathrm{AM}$ on $5 \mathrm{dpf}$. In addition to the final survivorship count on $5 \mathrm{dpf}$, we quantified incidences of pericardial edema and imaged larvae with a ZEISS SteREO Discovery.V8 Microscope equipped with a Jenoptik Gryphax Arktur camera. 


\subsection{Hair cell counts}

Wild type fish were crossed with the Tg(myo6b:eGFP-pA)vo68Tg line [33] to produce zebrafish larvae expressing green fluorescent protein in hair cells. Hair cell counts were done at the 0,50,100 and $200 \mu \mathrm{M}$ concentrations for Timeline One (6-hour intervals) and at all concentrations for Timeline Two (12-hour intervals). Hair cell counts for Timeline One did not include the 300 and $400 \mu \mathrm{M}$ concentrations due to high larval mortality at later treatments. Additionally, hair cells with functional transduction channels were labeled with $3 \mu$ M FM 4-64 (Thermo Fisher Scientific) in E3 embryo media for 30 seconds, followed by rinses in E3. Figure 1A outlines the imaging time points for both timelines. For both cases, the first round of imaging was done after an hour of recovery time following the first neomycin treatment (the morning of $3 \mathrm{dpf}$ ). The second round of imaging was done an hour before the second neomycin treatment (the afternoon/evening of $3 \mathrm{dpf}$ ). The third round of imaging was done after an hour of recovery time following the neomycin treatment on the morning of $4 \mathrm{dpf}$. The fourth and final round of imaging was done an hour before the next neomycin treatment on the afternoon/evening of $4 \mathrm{dpf}$. For all experiments, 6-9 larvae from treated and untreated groups were randomly chosen for imaging. Live larvae were mounted in $1.2 \%$ low melting point agarose (IBI Scientific) dissolved in E3. The L1, MI1 and O2 neuromasts were imaged on a Zeiss LSM 800 confocal. After imaging, larvae were freed from the agarose and returned to their respective control or experimental groups. GFP and FM 4-64 cell counts were performed using the Z-stack data.

\subsection{Statistics}

Statistical analyses were done using the R stats and betareg packages in RStudio $[45,46]$. P-values of less than 0.05 were considered statistically significant. Plots were made with ggplot2 [47].

Supplementary Materials: The following are available online at www.mdpi.com/xxx/s1, Figure S1, Tables S1-S4.

Author Contributions: Conceptualization, A.V. and T.E.; Investigation, A.V.; Visualization, A.V.; Formal analysis, A.V.; Resources, T.E.; Writing-Original Draft Preparation, A.V.; Writing-Manuscript editing, T.E.; Supervision, T.E.; Funding Acquisition, T.E. Both authors have read and agreed to the published version of the manuscript.

Funding: This work was funded by East Carolina University's Division of Research, Economic Development and Engagement (REDE) and The Natural Science and Engineering Research Council (NSERC) of Canada Discovery Grant RGPIN-2021-03166 to T.E.

Data Availability Statement: The raw data generated in the study are available upon request from the corresponding author.

Acknowledgments: The authors thank Albert Tucci for assisting with statistical analysis and data visualization. We also thank members of the Erickson lab for their assistance with animal husbandry.

Conflicts of Interest: The authors declare no conflict of interest.

\section{References}

1. Mogdans, J. Sensory ecology of the fish lateral-line system: Morphological and physiological adaptations for the perception of hydrodynamic stimuli. Journal of Fish Biology 2019, 95, jfb.13966, doi:10.1111/jfb.13966.

2. Montgomery, J.C.; Baker, C.F. Lateral line and fish behavior. In The Senses: A Comprehensive Reference; Elsevier, 2020; pp. 133142.

3. Montgomery, J.C.; Baker, C.F.; Carton, A.G. The lateral line can mediate rheotaxis in fish. Nature 1997, 389, 960-963, doi:10.1038/40135. 
4. Baker, C.F.; Montgomery, J.C. The sensory basis of rheotaxis in the blind Mexican cave fish, Astyanax fasciatus. Journal of Comparative Physiology A: Sensory, Neural, and Behavioral Physiology 1999, 184, 519-527, doi:10.1007/s003590050351.

5. Suli, A.; Watson, G.M.; Rubel, E.W.; Raible, D.W. Rheotaxis in larval zebrafish is mediated by lateral line mechanosensory hair cells. PLoS ONE 2012, 7, e29727, doi:10.1371/journal.pone.0029727.

6. Coombs, S.; Finneran, J.J.; Conley, R.A. Hydrodynamic image formation by the peripheral lateral line system of the Lake Michigan mottled sculpin, Cottus bairdi. Philosophical Transactions of the Royal Society of London. Series B: Biological Sciences 2000, 355, 1111-1114, doi:10.1098/RSTB.2000.0649.

7. Stewart, W.J.; Cardenas, G.S.; McHenry, M.J. Zebrafish larvae evade predators by sensing water flow. Journal of Experimental Biology 2013, 216, 388-398, doi:10.1242/jeb.072751.

8. Coffin, A.B.; Ramcharitar, J. Chemical ototoxicity of the fish inner ear and lateral line. In Advances in Experimental Medicine and Biology; Springer, Cham, 2016; Vol. 877, pp. 419-437.

9. Ton, C.; Parng, C. The use of zebrafish for assessing ototoxic and otoprotective agents. Hearing Research 2005, 208, 79-88, doi:10.1016/j.heares.2005.05.005.

10. Linbo, T.L.; Stehr, C.M.; Incardona, J.P.; Scholz, N.L. Dissolved copper triggers cell death in the peripheral mechanosensory system of larval fish. Environmental toxicology and chemistry 2006, 25, 597-603, doi:10.1897/05-241r.1.

11. Ou, H.C.; Raible, D.W.; Rubel, E.W. Cisplatin-induced hair cell loss in zebrafish (Danio rerio) lateral line. Hearing Research 2007, 233, 46-53, doi:10.1016/j.heares.2007.07.003.

12. Williams, J.A.; Holder, N. Cell turnover in neuromasts of zebrafish larvae. Hearing Research 2000, 143, 171-181, doi:10.1016/S0378-5955(00)00039-3.

13. Harris, J.A.; Cheng, A.G.; Cunningham, L.L.; MacDonald, G.; Raible, D.W.; Rubel, E.W. Neomycin-induced hair cell death and rapid regeneration in the lateral line of zebrafish (Danio rerio). JARO - Journal of the Association for Research in Otolaryngology 2003, 4, 219-234, doi:10.1007/s10162-002-3022-x.

14. Olivari, F.A.; Hernández, P.P.; Allende, M.L. Acute copper exposure induces oxidative stress and cell death in lateral line hair cells of zebrafish larvae. Brain Research 2008, 1244, 1-12, doi:10.1016/J.BRAINRES.2008.09.050.

15. Owens, K.N.; Cunningham, D.E.; Macdonald, G.; Rubel, E.W.; Raible, D.W.; Pujol, R. Ultrastructural analysis of aminoglycosideinduced hair cell death in the zebrafish lateral line reveals an early mitochondrial response. Journal of Comparative Neurology 2007, doi:10.1002/cne.21345.

16. Esterberg, R.; Linbo, T.; Pickett, S.B.; Wu, P.; Ou, H.C.; Rubel, E.W.; Raible, D.W. Mitochondrial calcium uptake underlies ROS generation during aminoglycoside-induced hair cell death. Journal of Clinical Investigation 2016, 126, 3556-3566, doi:10.1172/JCI84939.

17. Sha, S.-H.; Schacht, J. Stimulation of free radical formation by aminoglycoside antibiotics. Hearing Research 1999, 128, 112-118, doi:10.1016/S0378-5955(98)00200-7.

18. Hirose, K.; Hockenbery, D.M.; Rubel, E.W. Reactive oxygen species in chick hair cells after gentamicin exposure in vitro. Hearing Research 1997, 104, 1-14, doi:10.1016/S0378-5955(96)00169-4.

19. Forge, A.; Schacht, J. Aminoglycoside antibiotics. Audiology \& neuro-otology 2000, 5, 3-22, doi:10.1159/000013861.

20. Kros, C.J.; Steyger, P.S. Aminoglycoside- and cisplatin-induced ototoxicity: Mechanisms and otoprotective strategies. Cold Spring Harbor Perspectives in Medicine 2018, 9, a033548, doi:10.1101/CSHPERSPECT.A033548.

21. Murakami, S.L.; Cunningham, L.L.; Werner, L.A.; Bauer, E.; Pujol, R.; Raible, D.W.; Rubel, E.W. Developmental differences in susceptibility to neomycin-induced hair cell death in the lateral line neuromasts of zebrafish (Danio rerio). Hearing Research 2003, 186, 47-56, doi:10.1016/S0378-5955(03)00259-4.

22. Santos, F.; MacDonald, G.; Rubel, E.W.; Raible, D.W. Lateral line hair cell maturation is a determinant of aminoglycoside susceptibility in zebrafish (Danio rerio). Hearing Research 2006, 213, 25-33, doi:10.1016/J.HEARES.2005.12.009. 
23. Ghysen, A.; Dambly-Chaudière, C. The lateral line microcosmos. Genes E Development 2007, 21, 2118-2130, doi:10.1101/GAD.1568407.

24. Ledent, V. Postembryonic development of the posterior lateral line in zebrafish. Development (Cambridge, England) 2002, 129, 597-604.

25. Metcalfe, W.K.; Kimmel, C.B.; Schabtach, E. Anatomy of the posterior lateral line system in young larvae of the zebrafish. Journal of Comparative Neurology 1985, 233, 377-389, doi:10.1002/cne.902330307.

26. Raible, D.W.; Kruse, G.J. Organization of the lateral line system in embryonic zebrafish. Journal of Comparative Neurology 2000, 421, 189-198, doi:10.1002/(SICI)1096-9861(20000529)421:2<189::AID-CNE5>3.0.CO;2-K.

27. Mackenzie, S.M.; Raible, D.W. Proliferative regeneration of zebrafish lateral line hair cells after different ototoxic insults. PLoS ONE 2012, 7, e47257, doi:10.1371/journal.pone.0047257.

28. Alharazneh, A.; Luk, L.; Huth, M.; Monfared, A.; Steyger, P.S.; Cheng, A.G.; Ricci, A.J. Functional hair cell mechanotransducer channels are required for aminoglycoside ototoxicity. PloS one 2011, 6, e22347, doi:10.1371/journal.pone.0022347.

29. Owens, K.N.; Coffin, A.B.; Hong, L.S.; Bennett, K.O.; Rubel, E.W.; Raible, D.W. Response of mechanosensory hair cells of the zebrafish lateral line to aminoglycosides reveals distinct cell death pathways. Hearing Research 2009, 253, 32-41, doi:10.1016/j.heares.2009.03.001.

30. Seiler, C.; Nicolson, T. Defective Calmodulin-dependent rapid apical endocytosis in zebrafish sensory hair cell mutants. Journal of Neurobiology 1999, 41, 424-434, doi:10.1002/(SICI)1097-4695(19991115)41:3<424::AID-NEU10>3.0.CO;2-G.

31. Hernández, P.P.; Olivari, F.A.; Sarrazin, A.F.; Sandoval, P.C.; Allende, M.L. Regeneration in zebrafish lateral line neuromasts: Expression of the neural progenitor cell marker Sox 2 and proliferation-dependent and-independent mechanisms of hair cell renewal. Developmental Neurobiology 2007, 67, 637-654, doi:10.1002/dneu.20386.

32. McHenry, M.J.; Feitl, K.E.; Strother, J.A.; van Trump, W.J. Larval zebrafish rapidly sense the water flow of a predator's strike. Biology Letters 2009, 5, 477-479, doi:10.1098/rsbl.2009.0048.

33. Erickson, T.; Pacentine, I. v.; Venuto, A.; Clemens, R.; Nicolson, T. The lhfpl5 ohnologs $l h f p l 5 a$ and $l h f p l 5 b$ are required for mechanotransduction in distinct populations of sensory hair cells in zebrafish. Frontiers in Molecular Neuroscience 2020, 12, 320, doi:10.3389/fnmol.2019.00320.

34. Kindt, K.S.; Finch, G.; Nicolson, T. Kinocilia mediate mechanosensitivity in developing zebrafish hair cells. Developmental Cell 2012, 23, 329-341, doi:10.1016/j.devcel.2012.05.022.

35. Meyers, J.R.; MacDonald, R.B.; Duggan, A.; Lenzi, D.; Standaert, D.G.; Corwin, J.T.; Corey, D.P. Lighting up the senses: FM1-43 loading of sensory cells through nonselective ion channels. Journal of Neuroscience 2003, 23, 4054-4065, doi:10.1523/jneurosci.2310-04054.2003.

36. Han, J.; Liu, K.; Wang, R.; Zhang, Y.; Zhou, B. Exposure to cadmium causes inhibition of otolith development and behavioral impairment in zebrafish larvae. Aquatic Toxicology 2019, 214, 105236, doi:10.1016/J.AQUATOX.2019.105236.

37. Hansen, J.A.; Rose, J.D.; Jenkins, R.A.; Gerow, K.G.; Bergman, H.L. Chinook salmon (Oncorhynchus tshawytscha) and rainbow trout (Oncorhynchus mykiss) exposed to copper: Neurophysiological and histological effects on the olfactory system. Environmental Toxicology and Chemistry 1999, 18, 1979-1991, doi:10.1002/etc.5620180917.

38. Xu, J.; Zhang, R.; Zhang, T.; Zhao, G.; Huang, Y.; Wang, H.; Liu, J.-X. Copper impairs zebrafish swimbladder development by down-regulating Wnt signaling. Aquatic Toxicology 2017, 192, 155-164, doi:10.1016/J.AQUATOX.2017.09.018.

39. Carreau, N.D.; Pyle, G.G. Effect of copper exposure during embryonic development on chemosensory function of juvenile fathead minnows (Pimephales promelas). Ecotoxicology and Environmental Safety 2005, 61, 1-6, doi:10.1016/j.ecoenv.2004.10.008.

40. Han, E.; Ho Oh, K.; Park, S.; Chan Rah, Y.; Park, H.C.; Koun, S.; Choi, J. Analysis of behavioral changes in zebrafish (Danio rerio) larvae caused by aminoglycoside-induced damage to the lateral line and muscles. NeuroToxicology 2020, 78, 134-142, doi:10.1016/j.neuro.2020.03.005. 
41. Cruz, I.A.; Kappedal, R.; Mackenzie, S.M.; Hailey, D.W.; Hoffman, T.L.; Schilling, T.F.; Raible, D.W. Robust regeneration of adult zebrafish lateral line hair cells reflects continued precursor pool maintenance. Developmental Biology 2015, 402, 229-238, doi:10.1016/J.YDBIO.2015.03.019.

42. Hardy, K.; Amariutei, A.E.; de Faveri, F.; Hendry, A.; Marcotti, W.; Ceriani, F. Functional development and regeneration of hair cells in the zebrafish lateral line. The Journal of Physiology 2021, 599, 3913-3936, doi:10.1113/JP281522.

43. Carrillo, A.; McHenry, M.J. Zebrafish learn to forage in the dark. Journal of Experimental Biology 2016, 219, 582-589, doi:10.1242/jeb.128918.

44. Westerfield, M. The Zebrafish Book. A Guide for the Laboratory Use of Zebrafish (Danio Rerio).; 4th ed.; University of Oregon: Eugene OR, 2000;

45. RStudio Team RStudio: Integrated development environment for R 2018.

46. R Core Team R: A language and environment for statistical computing 2019.

47. Wickham, H. ggplot2. Wiley Interdisciplinary Reviews: Computational Statistics 2011, 3, 180-185, doi:10.1002/wics.147. 